\title{
FEMTOSECOND LIBS AND SECOND HARMONIC GENERATION TECHNIQUES FOR DETECTION OF CHEMICAL AND BIOLOGICAL AGENTS
}

\author{
Dennis R. Alexander \\ Professor of Electrical Engineering \\ Center for Electro-Optics \\ University of Nebraska - Lincoln
}

\begin{abstract}
The following scientific paper was presented at the Merrill conference by Prem Paul, Vice Chancellor for Research, as an example of the unique expertise available at the University of Nebraska - Lincoln. It was prepared by Dennis R. Alexander at the Center for Electro-Optics.
\end{abstract}

\section{Introduction}

Lasers generating ultrashort light pulses from about 5 to 100's of femtoseconds are now available to researchers for carrying out femtosecond laser induced breakdown spectroscopy. Recent technological advances in ultrafast technologies have resulted in the generation of light packets consisting of only a few cycles of the electric and magnetic fields. The spatial extension of these wave packets along the direction of propagation is limited to a few times the wavelength of the radiation $(\sim 0.5-1 \mu \mathrm{m}$ in the visible and near-infrared spectral range). On the other hand, a $100 \mathrm{fs}$ pulse has a packet length of $30 \mu \mathrm{m}$. When using diffraction limited parabolic mirror for focusing and moderate pulse energies of one microjoule, peak intensities at the focal spot of over $10^{15} \mathrm{~W} / \mathrm{cm}^{2}$ can be achieved. The corresponding amplitude of the electric field at these intensities approaches $10^{9} \mathrm{~V} / \mathrm{cm}^{2}$. These field strengths are high enough to trigger optical field ionization. Hence, detachment of the first electron is completed at substantially higher field strength and the optical-field ionization rate becomes comparable to the laser field oscillation frequency. The released electrons gain unprecedented kinetic energies (up to and beyond the keV level) during the first field oscillation cycle following their detachment, and a substantial fraction of the ionization occurs during one cycle of light. In comparison, long pulsed laser systems containing many field oscillation cycles depletes the atomic ground state. The above linear and nonlinear processes result in very precise thresholds for plasma formation since femtosecond interactions produce their own source of free electrons to initiate the plasma formation process. Longer nanosecond pulses produce breakdown at less defined thresholds. This paper discusses some preliminary results into the use of femtosecond lasers for performing FLIBS and the second harmonic detection of chemical and biological warfare agents. 


\section{Experimental Facilities}

The Center for Electro-Optics at the University of Nebraska - Lincoln has three femtosecond laser systems that can be used for FLIBS and second order harmonic generation. The first system is a Spectra Physics Millennium pumped Tsunami oscillator that is then amplified with an Applied Photonics Industries $\mathrm{Nd}$ :YLF laser. This system typically produces 100's of fs pulses at a center wavelength of $795 \mathrm{~nm}$. The system is capable of producing $900 \mathrm{~mJ}$ pulses at a frequency that can be selected from 1 to $1000 \mathrm{~Hz}$. The second laser system is a FemtoSource Compact manufactured by FemtoLasers, Viena, Austria. This system is capable of producing $<10 \mathrm{fs}$ pulses. The laser operates at a wavelength centered at $795 \mathrm{~nm}$ and at a frequency of $75 \mathrm{MHz}$. The third femtosecond laser system is a Spectra Physics Millennium Pumped Spitfire system that is pumped with a Kapteyn-Murnane oscillator. This system produces $<35 \mathrm{fs}$ pulses at a frequency of $1000 \mathrm{~Hz}$ with $700 \mathrm{~mJ}$ of pulse energy. Plasmas as well as second harmonic generation are produced by focusing the laser pulses on the material of interest using both lens and parabolic mirrors. Femtosecond produced plasmas and second order harmonic generation are collected on an Instruments SA Optical Multi-channel Analyzer (OMA). The detection device is a Princeton Instruments gated CCD array, Model ICCD-124MG-E, with a 6-phase array.

\section{Results}

In many LIBS applications for the detection of chemical and biological agents there is a need to limit the degree of damage to the material of interest. In addition there is a need to be able to detect these species at very low concentrations. Fig. 1 demonstrates the chemical detection of the chemical keratin in the human hair. The spot on the middle right appears distinctly blue and is the second order emission from the keratin molecule. The hair strand is being illuminated at $800 \mathrm{~nm}$ and the blue emission occurs at $400 \mathrm{~nm}$.

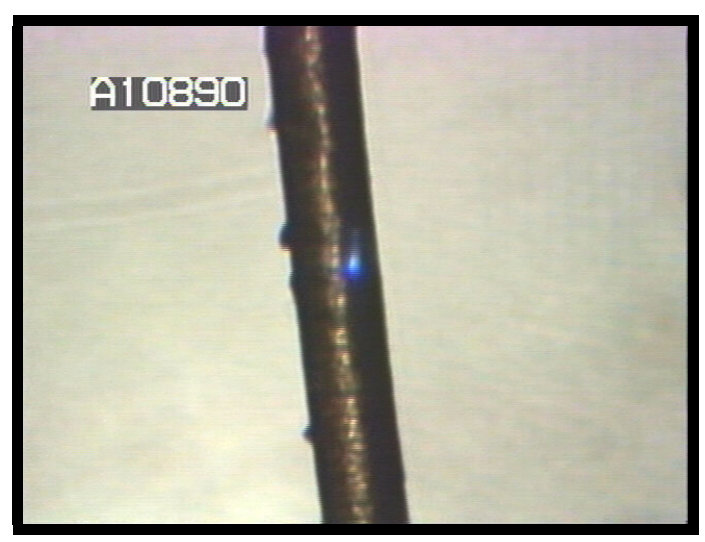

Fig. 1. Second harmonic generation blue emission (SHG) $(\lambda=400 \mathrm{~nm})$ is visible in the image during femtosecond laser interaction $(\lambda=800 \mathrm{~nm})$ on a human hair. A CCD camera captures the blue emission for demonstrating this capability to detect chemical agents 
Because of the unique breakdown thresholds offered by the femtosecond laser, it is possible to produce very small damage sites. These damage sites can be as small as about 1 micron. Shown in Fig. 2 is a one micron hole drilled in a silicon wafer. In this application, FLIBS was used to investigate the penetration of a beam to various layers in the silicon chip. By monitoring the spectral components of the emission, it is possible to determine which layer in the chip one has reached. This has important applications in chip failure analysis.

One of the problems with using longer pulsed lasers is that they produce plasmas containing large continuum components. The usual mode of performing LIBS is to wait for these components to die out before the collection of the longer lived atomic emission lines. Fig. 3 compares the difference between femtosecond produced plasmas on an aluminum target and the nanosecond case. The advantage of the femtosecond spectrum is that the peaks emerge from the base line while in the nanosecond case the peaks appear on top of a broad continuum. Fig. 3 demonstrates the difference in the size of the damage region for nanosecond laser ablation as compared to that achieved for femtosecond ablation. Further information will be presented that relates the plasma formation and the damage to the unique way that plasmas are produced during femtosecond interactions. The advantages and disadvantages of using femtosecond LIBS will be presented in greater detail.

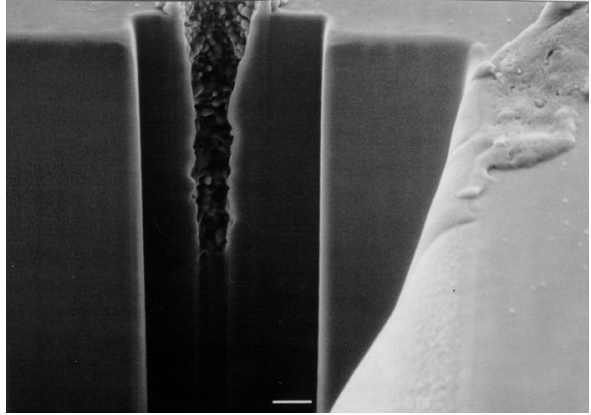

100 pulses, $44.2 \mathrm{~nJ}$

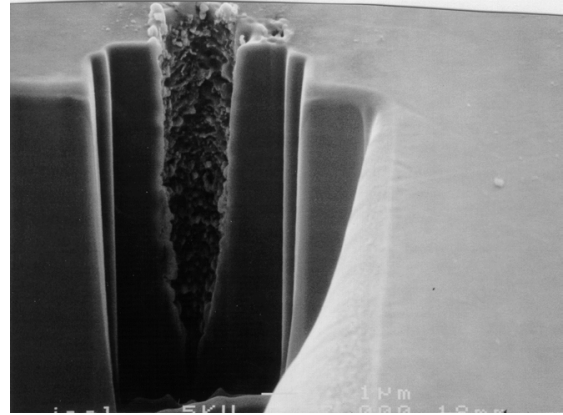

1000 pulses, $44.2 \mathrm{~nJ}$

Fig. 2 Femtosecond lasers allow less than the diffraction limit holes to be placed in materials. Another advantage is that high aspect ratio holes can be drilled in materials. The material in these images is an AMD silicon chip. 

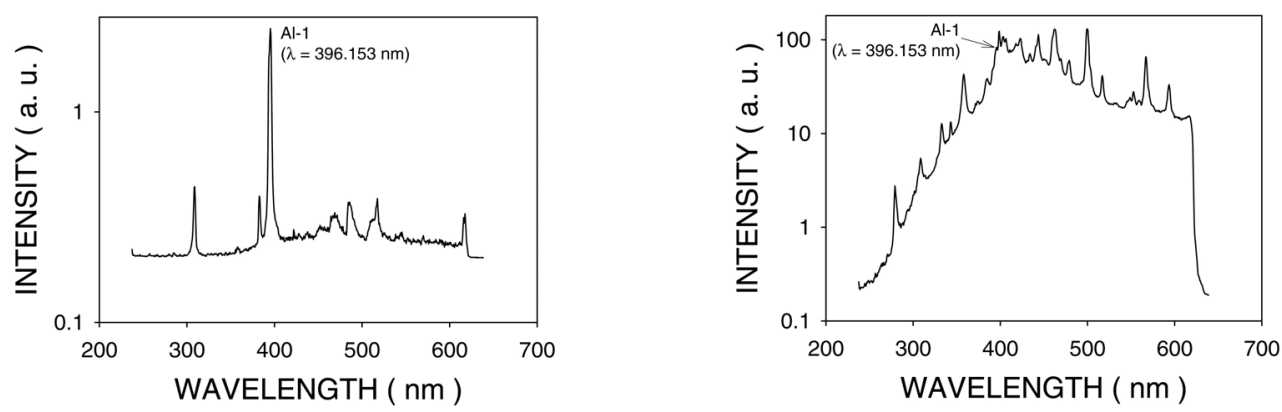

Fig. 3 Comparison of femtosecond produced plasma emission lines (left) or aluminum film and those obtained from a nanosecond formed plasma (right). The broad background emission is not present in the femtosecond case. This makes the detection of chemical and biological agents at low concentrations easier since the peaks come directly out of the baseline.
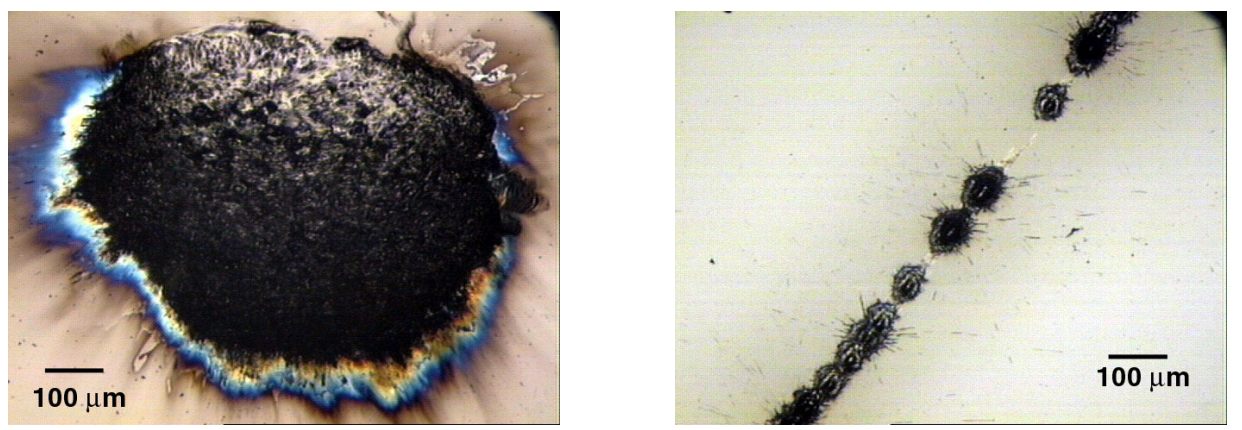

Fig. 4. These images show the difference between the size of the damage spot for the nanosecond (left) and femtosecond (right) of aluminum films 